\title{
Influence of spin-transfer torque on thermally activated ferromagnetic resonance excitations in magnetic tunnel junctions
}

\author{
S. Petit, ${ }^{1}$ N. de Mestier, ${ }^{1}$ C. Baraduc, ${ }^{1}$ C. Thirion, ${ }^{1}$ Y. Liu, ${ }^{2}$ M. Li ${ }^{2}$ P. Wang, ${ }^{2}$ and B. Dieny ${ }^{1}$ \\ ${ }^{1}$ SPINTEC, CEA/CNRS/UJF/INPG; CEA/INAC, 17 rue des Martyrs, 38054 Grenoble Cedex, France \\ ${ }^{2}$ Headway Technologies, 6785 Hillview Drive, Milpitas, California 95035, USA \\ (Received 11 June 2008; revised manuscript received 19 October 2008; published 18 November 2008)
}

\begin{abstract}
Voltage noise measurements on magnetic tunnel junctions show that thermal fluctuations of the magnetization are either amplified or quenched by subcritical spin-transfer torque depending on the current direction. We present an analytical model that describes the dependence of thermally activated ferromagnetic resonance on bias current. The evolution of the peak amplitude and linewidth with the applied current is directly related to the longitudinal torque, whereas the shift of the resonance frequency is sensitive to the transverse torque. Both spin torque terms are independently extracted from the measured noise spectra. Our results support the general idea that it is more pertinent to describe spin torque in terms of voltage rather than current in magnetic tunnel junctions.
\end{abstract}

DOI: 10.1103/PhysRevB.78.184420

PACS number(s): 05.40.-a, 75.47. $-\mathrm{m}, 75.75 .+\mathrm{a}, 76.50 .+\mathrm{g}$

\section{INTRODUCTION}

Since its first theoretical description, ${ }^{1,2}$ the phenomenon of spin-transfer torque has received considerable attention. This phenomenon corresponds to the reciprocal effect of spin filtering and can be described as a direct transfer of angular momentum from the spin-polarized conduction electrons to the local magnetization. At bias current larger than the critical current, spin torque leads either to magnetization reversal ${ }^{3,4}$ or to steady-state precession. ${ }^{5-7}$ Both effects are widely studied in relation to potential applications such as magnetic random access memory (MRAM) writing or radiofrequency oscillators. Nevertheless, in the subcritical regime (i.e., at current density much below the critical current density for spin-torque switching) where there is no macroscopic signature of this effect, we have shown that spin-transfer torque influences the magnetization thermal fluctuations. ${ }^{8}$ In this paper, we present a detailed analysis of this phenomenon. In Sec. II, an analytical macrospin model is developed in the framework of the linear-response theory. First we calculate the magnetic noise power spectral density in the absence of spin-transfer torque. Then we reconsider the magnetization dynamics in the presence of spin torque in the subcritical regime when the system remains close to thermodynamic equilibrium. The experimental technique used to measure voltage noise is described in Sec. III, and the electronic transport in our $\mathrm{MgO}$ based magnetic tunnel junctions is reported in Sec. IV. Finally the influence of spin-transfer torque on magnetic fluctuations is analyzed in Sec. V in relation with our analytical model. By contrast to our previous work on alumina tunnel junctions, the larger signals obtained in $\mathrm{MgO}$ tunnel junctions enable us to clearly identify a center mode peak in the noise spectra (for which the magnetization dynamics amplitude is larger in the center of the sample and weaker on the edges). This mode was thoroughly studied since it should be more adequately described by our simple macrospin model. In this paper, all the parameters of the model are independently extracted either from the field or from the current dependence of the magnetic noise. The longitudinal and transverse spin torque terms are estimated from this analysis and their dependences on current and on voltage are discussed. This study confirms Slonczewski's ${ }^{9}$ prediction that a critical voltage is a more appropriate concept than a critical current for tunnel junctions.

\section{MODEL}

The influence of spin torque on magnetization fluctuations is modeled in the framework of the linear-response theory in the macrospin approximation in order to derive a general susceptibility tensor. First well-known results on magnetic noise ${ }^{10}$ in the absence of spin torque are presented so that the reader becomes familiar with our notations and calculation methods. Then we introduce the longitudinal and transverse spin-transfer torque terms and analyze how they, respectively, affect the susceptibility.

\section{A. Magnetic fluctuations in the absence of spin torque}

Let us consider a continuous ferromagnetic film with the $\hat{x}$ axis perpendicular to the plane, subjected to a static magnetic field applied along the $\hat{z}$ direction. In the following calculations, we neglect magnetocrystalline anisotropy without any loss of generality. Magnetization dynamics is assumed to follow the Landau-Lifshitz-Gilbert (LLG) equation,

$$
\frac{d \vec{M}}{d t}=-\gamma_{0} \vec{M} \times \vec{H}_{\mathrm{eff}}+\frac{\alpha}{M_{s}} \vec{M} \times \frac{d \vec{M}}{d t},
$$

where $\gamma_{0}, \alpha, M_{s}$, and $\vec{H}_{\text {eff }}$ are, respectively, the gyromagnetic ratio, the Gilbert damping constant, the saturation magnetization, and the effective field. The effective field is the sum of the applied field $\vec{H}=H \hat{z}$ and the demagnetizing field $-4 \pi M_{x} \hat{x}$. In order to describe magnetic noise, we consider the Langevin approach where the microscopic interactions with the thermal bath are described by a random field. Following Brown's ${ }^{11}$ approach, this random field is supposed stationary and isotropic with a Gaussian distribution and satisfying the following correlation conditions: $\left\langle h_{i}(t) h_{j}(t+\tau)\right\rangle$ $\propto \delta_{i, j} \delta(\tau)$. The Langevin field acts as a supplementary exter- 
nal magnetic field and contributes to the effective field. Then the dynamics [Eq. (1)] can be projected on the three axes $\hat{x}$, $\hat{y}$, and $\hat{z}$. For small fluctuations around the equilibrium position, the transverse components of magnetization are negligible compared to the saturation magnetization. Therefore we assume $M_{z} \approx M_{s}$ and focus on the transverse magnetization $\vec{m}$ whose dynamics is described by coupled equations written in the following matrix form: ${ }^{12,13}$

$$
\left[\begin{array}{cc}
\alpha & -1 \\
1 & \alpha
\end{array}\right] \frac{d \vec{m}}{d t}+\gamma_{0}\left[\begin{array}{cc}
\tilde{H} & 0 \\
0 & H
\end{array}\right] \vec{m}=\gamma_{0} M_{s} \vec{h}
$$

where $\widetilde{H}=4 \pi M_{s}+H$. As the system is in static equilibrium, it can be described by the linear-response theory $\vec{m}(\omega)$ $=\underline{\chi}(\omega) \vec{h}(\omega) \cdot \underline{\chi}$ is the susceptibility tensor and is immediately deduced from Eq. (2),

$$
\begin{aligned}
& \underline{\chi}(\omega)=\gamma_{0} M_{s}\left(\gamma_{0}\left[\begin{array}{cc}
\tilde{H} & 0 \\
0 & H
\end{array}\right]-i \omega\left[\begin{array}{cc}
\alpha & -1 \\
1 & \alpha
\end{array}\right]\right)^{-1}, \\
& \underline{\chi}(\omega)=\frac{\gamma_{0} M_{s}}{\tilde{\omega}^{2}-i \omega \Lambda}\left[\begin{array}{cc}
\gamma_{0} H-i \omega \alpha & -i \omega \\
i \omega & \gamma_{0} \tilde{H}-i \omega \alpha
\end{array}\right],
\end{aligned}
$$

with the following notations:

$$
\begin{gathered}
\omega_{0}^{2}=\gamma_{0}^{2} \tilde{H} H, \\
\widetilde{\omega}^{2}=\omega_{0}^{2}-\left(1+\alpha^{2}\right) \omega^{2}, \\
\Lambda=\gamma_{0} \alpha(\tilde{H}+H) .
\end{gathered}
$$

As the susceptibility is a tensor of complex variables, it is possible to write its real $\underline{\chi}^{\prime}$ and imaginary $\underline{\chi}^{\prime \prime}$ parts separately;

$$
\begin{gathered}
\underline{\chi}^{\prime}(\omega)=\frac{\gamma_{0} M_{s}}{\widetilde{\omega}^{4}+\omega^{2} \Lambda^{2}}\left[\begin{array}{cc}
\gamma_{0} H \widetilde{\omega}^{2}+\alpha \Lambda \omega^{2} & \Lambda \omega^{2} \\
-\Lambda \omega^{2} & \gamma_{0} \tilde{H} \widetilde{\omega}^{2}+\alpha \Lambda \omega^{2}
\end{array}\right], \\
\underline{\chi}^{\prime \prime}(\omega)=\frac{\gamma_{0} M_{s} \omega}{\widetilde{\omega}^{4}+\omega^{2} \Lambda^{2}}\left[\begin{array}{cc}
\gamma_{0} H \Lambda-\alpha \widetilde{\omega}^{2} & -\widetilde{\omega}^{2} \\
\widetilde{\omega}^{2} & \gamma_{0} \tilde{H} \Lambda-\alpha \widetilde{\omega}^{2}
\end{array}\right] .
\end{gathered}
$$

It is known as the fluctuation-dissipation theorem (FDT) that the fluctuations power spectral density is linked to the dissipative part of the linear response. In the case of correlated variables, the fluctuation-dissipation theorem is written as $^{14}$

$$
\underline{S}_{\vec{m}}=\left[\begin{array}{cc}
S_{m_{x} m_{x}} & S_{m_{x} m_{y}} \\
S_{m_{y} m_{x}} & S_{m_{y} m_{y}}
\end{array}\right]=\frac{4 k_{B} T}{\mu_{0} V} \frac{1}{\omega}\left[\begin{array}{cc}
\chi_{x x}^{\prime \prime} & \frac{1}{i} \chi_{x y}^{\prime} \\
\frac{1}{i} \chi_{y x}^{\prime} & \chi_{y y}^{\prime \prime}
\end{array}\right],
$$

where $S_{m_{i} m_{i}}$ represents the power spectral density of the fluctuating magnetization component $m_{i}$. It will be noted $S_{i i}$ in the rest of the paper. If we now calculate the different susceptibility coefficients in the matrix [Eq. (10)], we observe that they all show a peak at the ferromagnetic resonance frequency (FMR) with very different amplitudes. The largest value is obtained for $\chi_{y y}^{\prime \prime}$. Moreover the cross-correlated terms cannot be measured since $\chi_{x y}^{\prime}=-\chi_{y x}^{\prime}$. We therefore conclude that in-plane magnetic fluctuations $S_{y y}$ are the dominant contribution to the noise. This result was expected since out-of-plane fluctuations are limited by a strong demagnetizing factor. A closer look at $S_{y y}$ expression gives some physical insight. In particular it is possible to determine the resonance frequency $\omega_{r}$ where the peak amplitude is maximum. It is easily determined by calculating the frequency at which the first derivative of $S_{y y}$ is zero. When the damping factor $\alpha$ can be neglected, this expression reduces to the well-known Kittel formula $\omega_{r}^{2} \approx \gamma_{0}^{2} \tilde{H} H$. Taking into account the fact that $4 \pi m_{s} \gg H$, this formula states that the resonance peak shifts toward higher frequencies in a nearly linear way when the applied field increases. This behavior can be explained by the higher-energy cost of fluctuations under larger magnetic field. For small damping factor, the power spectral density of magnetization fluctuations has a Lorentzian shape with a linewidth equal to $\Lambda$ and an amplitude inversely proportional to $\Lambda H$. Since $\widetilde{H} \gg H$, the peak linewidth $\Lambda$ is nearly constant and the peak amplitude varies nearly inversely to the applied field. The fact that the magnetic fluctuations peak becomes lower for increasing applied field is simply related to the increasing rigidity of the system.

\section{B. Magnetic fluctuations in the presence of spin torque}

Now we consider a multilayer structure with two ferromagnetic layers separated by a spacer. This structure corresponds to the active part of a spin valve or a magnetic tunnel junction. The magnetization of one of the ferromagnetic layer is supposed to be fixed (reference layer), whereas the magnetization of the other can move freely (free layer). The thin ferromagnetic layer that we considered in Sec. II A is now the free layer and is subjected to a perpendicular-toplane current. A negative current corresponds to the situation where electrons flow from the reference layer toward the free layer. In this case, the electron spins are first polarized by the reference layer and may exert a torque on the free layer magnetization through a direct transfer of angular momentum. Inversely, a positive current corresponds to electrons flowing from the free layer. Thus electrons that have their polarization opposite to the reference layer one are mostly reflected at the interface and are responsible for the torque on the free layer. This spin-transfer torque is phenomenologically written as the usual following expression: ${ }^{15}$

$$
\vec{T}=\gamma_{0}\left[\frac{a_{j}}{M_{s}} \vec{M} \times(\vec{M} \times \vec{p})+b_{j} \vec{M} \times \vec{p}\right] .
$$

The term proportional to $a_{j}$ is the Slonczewski spin torque term $^{2}$ that represents the in-plane torque where $\vec{p}$ is the polarization of the reference layer. By convention, the polarization $\vec{p}$ is defined as a unit vector along the $z$ axis $\vec{p}=\epsilon \hat{z}$, where $\epsilon=1$ in the parallel state $(\mathrm{P})$ and $\epsilon=-1$ in the antiparallel state (AP). The $b_{j}$ term is the transverse torque that acts as a supplementary magnetic field and will be hereafter re- 
ferred to as the fieldlike term. The magnetization dynamics is now described by a modified LLG equation taking into account the two spin torque terms

$$
\begin{aligned}
\frac{d \vec{M}}{d t}= & -\gamma_{0} \vec{M} \times\left(\vec{H}_{\mathrm{eff}}-b_{j} \vec{p}\right)+\frac{\alpha}{M_{s}} \vec{M} \times \frac{d \vec{M}}{d t} \\
& +\gamma_{0} \frac{a_{j}}{M_{s}} \vec{M} \times(\vec{M} \times \vec{p}) .
\end{aligned}
$$

Note that Eq. (12) is written here with the most widely used sign convention contrary to our previous paper. ${ }^{8}$ When the reference and free layers are in strictly parallel or antiparallel configuration, there is no spin-transfer torque effect on the equilibrium magnetization. However, spin-polarized electrons may exert a torque on the free layer fluctuating magnetization. This particular case with unaffected equilibrium magnetization is considered in the following. When the system is subjected to a dc bias current and to a random thermal field $\vec{h}$, the dynamics of the transverse magnetization is described by the following equation:

$$
\left[\begin{array}{cc}
\alpha & -1 \\
1 & \alpha
\end{array}\right] \frac{d \vec{m}}{d t}+\gamma_{0}\left[\begin{array}{cc}
\tilde{H}-\epsilon b_{j} & \epsilon a_{j} \\
-\epsilon a_{j} & H-\epsilon b_{j}
\end{array}\right] \vec{m}=\gamma_{0} M_{s} \vec{h} .
$$

In the linear-response approximation the susceptibility matrix has the same expression as in Eq. (4) with replacing $H$ by $H_{j}, \widetilde{H}$ by $\widetilde{H}_{j}, \omega_{0}$ by $\omega_{j}, \widetilde{\omega}^{2}$ by $\widetilde{\omega}_{j}^{2}$, and $\Lambda$ by $\Lambda_{j}$ with the following definitions:

$$
\begin{gathered}
H_{j}=H-\epsilon b_{j}, \\
\widetilde{H}_{j}=4 \pi M_{s}+H_{j}, \\
\omega_{j}^{2}=\gamma_{0}^{2}\left[\widetilde{H}_{j} H_{j}+a_{j}^{2}\right], \\
\widetilde{\omega}_{j}^{2}=\omega_{j}^{2}-\left(1+\alpha^{2}\right) \omega^{2}, \\
\Lambda_{j}=\gamma_{0}\left[\alpha\left(\widetilde{H}_{j}+H_{j}\right)-2 \epsilon a_{j}\right],
\end{gathered}
$$

From the susceptibility we may express the power spectral density of magnetization fluctuations, provided the fluctuation-dissipation theorem still applies. Since the system is subjected to an external torque due to the bias current, the system is no more at thermodynamic equilibrium. However, the FDT is widely used close to the equilibrium or in stationary states when the rate of relaxation is not really affected by the external forces applied to the system. In our case, this means that it is reasonable to assume that the FDT is still valid in the subcritical regime when the bias current is much lower than the critical current at which magnetization starts either to switch or to precess. From the following dynamics equation, it is now possible to define a qualitative criterion for which spin-transfer torque is only a small perturbation to the equilibrium;

$$
\frac{d \vec{m}}{d t}=-\frac{\gamma_{0}}{1+\alpha^{2}}\left[\begin{array}{cc}
\alpha \tilde{H}_{j}-\epsilon a_{j} & H_{j}+\alpha \epsilon a_{j} \\
-\widetilde{H}_{j}-\alpha \epsilon a_{j} & \alpha H_{j}-\epsilon a_{j}
\end{array}\right] \vec{m} .
$$

The solution of this dynamics equation is given by the complex conjugated eigenvalues $E$ and $E^{*}$ of the matrix. Here,

$$
E=\frac{-\Lambda_{j}}{1+\alpha^{2}}\left(\frac{1}{2}-\frac{i}{2} \sqrt{4\left(1+\alpha^{2}\right) \frac{\omega_{j}^{2}}{\Lambda_{j}^{2}}-1}\right) .
$$

Considering the expression of $\Lambda_{j}$, it is easy to show that these eigenvalues are not sensitively modified when $2 a_{j}$ $\ll \alpha(\widetilde{H}+H)$. The same criterion can also be obtained by statistical physics calculations. So when dealing with experimental data, we will a posteriori verify that this criterion is fulfilled. As in-plane transverse magnetization fluctuations are the major contribution to the noise in the FMR frequency range, we will specially study $S_{y y}$ which is related to the imaginary susceptibility $\chi_{y y}^{\prime \prime}$. The power spectral density $S_{y y}$ is described by Eq. (10) where $\chi_{y y}^{\prime \prime} / \omega$ is

$$
\frac{\chi_{y y}^{\prime \prime}(\omega)}{\omega}=\gamma_{0} M_{s} \frac{\gamma_{0} \widetilde{H}_{j} \Lambda_{j}-\alpha \widetilde{\omega}_{j}^{2}}{\widetilde{\omega}_{j}^{4}+\omega^{2} \Lambda_{j}^{2}} .
$$

As we noticed in Sec. II A, $S_{y y}$ expression is still pseudoLorentzian with a linewidth equal to $\Lambda_{j}$ and a peak amplitude inversely proportional to $\Lambda_{j} H_{j}$. To the first order, the peak linewidth $\Lambda_{j}$ depends linearly on the spin torque term $a_{j}$ with a slope equal to $-2 \gamma_{0}$. Depending on the current sign the peak becomes either larger and narrower or broader and lower. In that respect the spin torque term acts as an antidamping or extradamping term. When the spin torque term exactly compensates the Gilbert damping, the linewidth becomes zero and the peak diverges. This situation is obtained at the critical current consequently defined by $j_{c} \approx \epsilon \alpha(H$ $+\widetilde{H}) / 2 a$, assuming a linear dependence of $a_{j}, a_{j}=a j$, and neglecting $b_{j}$. Nevertheless, we must be cautious with this extrapolation up to the critical current since our model is theoretically only valid close to the equilibrium when $j \ll j_{c}$. The bias current does not only affect the peak amplitude and its linewidth but also the resonance frequency itself, however in a far less dramatic way. An exact calculation of the resonance frequency $\omega_{r}$ of $S_{y y}$ gives the following expression:

$$
\omega_{r}^{2}=\frac{1}{1+\alpha^{2}}\left[\omega_{j}^{2}-\frac{\gamma_{0} \Lambda_{j} \tilde{H}_{j}}{\alpha}\left(1-\frac{1+\alpha \epsilon a_{j} / \tilde{H}_{j}}{\sqrt{1+\alpha^{2}}}\right)\right] .
$$

A good approximation of the resonance frequency can be written, with $\alpha \ll 1$ and $a_{j} \ll M_{s}$, as

$$
\begin{aligned}
\omega_{r}^{2} \approx & \gamma_{0}^{2}\left[H \widetilde{H}-\epsilon(H+\widetilde{H}) b_{j}-\frac{\alpha^{2}}{2} \widetilde{H}(\widetilde{H}+3 H)\right. \\
& \left.+\epsilon \alpha a_{j}(2 \widetilde{H}+H)\right] .
\end{aligned}
$$

To the first order the resonance frequency does not depend on the spin torque term $a_{j}$ but on the fieldlike term $b_{j}$. This observation is consistent with the fact that the resonance frequency is sensitive to magnetic field. So our simple model shows that the two spin torque terms $a_{j}$ and $b_{j}$ can be inde- 

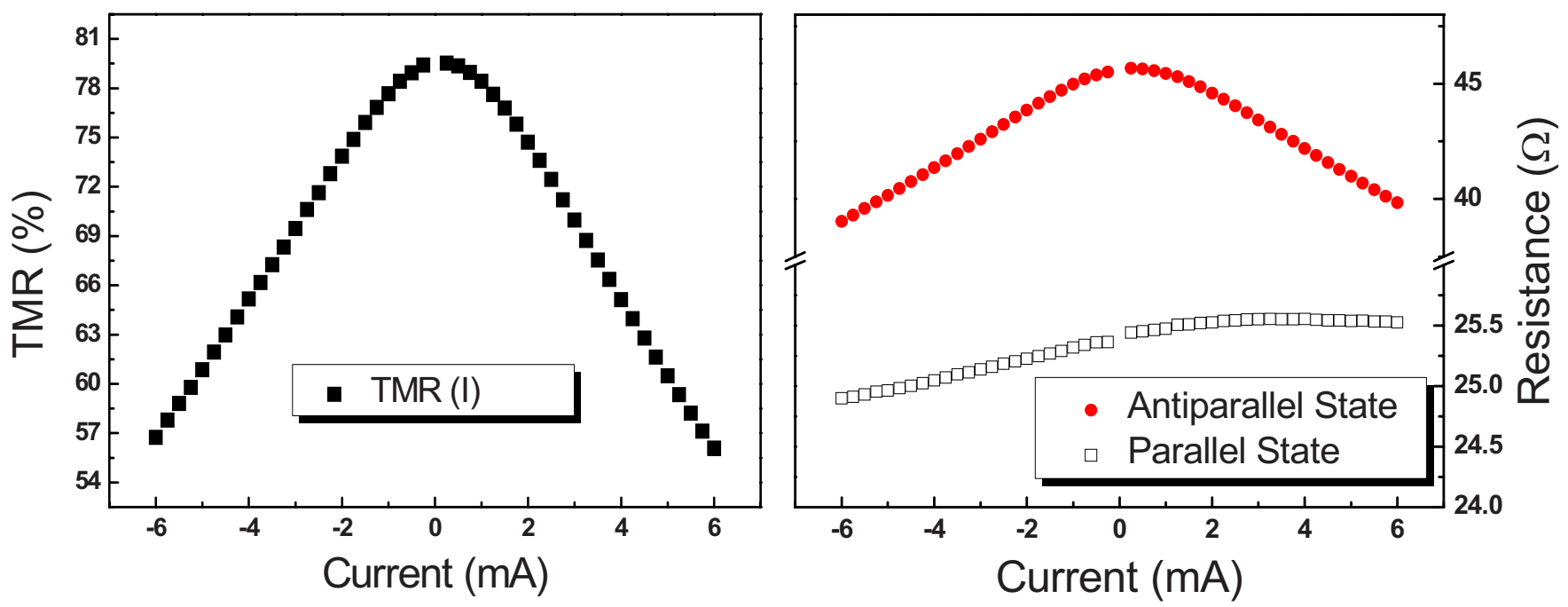

FIG. 1. (Color online) Left: tunnel magnetoresistance ratio as a function of bias current. Right: resistance in parallel and antiparallel states as a function of the applied current.

pendently extracted from the noise spectra at the ferromagnetic resonance.

\section{MEASUREMENT TECHNIQUE}

In a magnetoresistive device, magnetization fluctuations induce resistance fluctuations and therefore electrical noise. This voltage noise is measured in the gigahertz frequency range as a function of the external magnetic field and the dc current applied to the sample. To perform this measurement, the sample is placed in the gap of an electromagnet that produces an in-plane magnetic field. The sample is connected by a ground signal ground (GSG) rf probe and is fed with a dc current through the low-pass port of the bias T. The bias current is delivered by a Keithley 2400 source meter which noise is checked to be cut off by the bias T above 100 $\mathrm{MHz}$. Voltage noise spectra are measured through the highpass port of the bias T with a low noise preamplifier and an Agilent PSA E4440A spectrum analyzer. The data obtained are subsequently corrected in order to extract the exact value of the voltage noise produced by the tunnel junction. This data post-treatment is a three steps process. First the fluctuating voltage generated at the tunnel junction is not equal to the fluctuating voltage at the sample contact pads due to the extra impedances added by the contact lines. The relation between these two voltages is obtained by a de-embedding procedure described in the Appendix. Then the voltage at the contact pads is measured by the spectrum analyzer through a line composed of the rf probe, the bias $\mathrm{T}$, the preamplifier, and a cable. The transfer function of this line is determined as explained in the Appendix. Finally the magnetic noise is obtained by the following procedure: the noise power spectrum measured in the saturated magnetization state for each bias current is subtracted from the data in order to remove the background Johnson noise, the amplifier noise, and the shot noise as shown in Sec. IV B.

\section{TRANSPORT PROPERTIES}

We studied sputtered magnetic tunnel junctions with magnesium oxide tunnel barrier developed for read head appli- cations. These junctions are composed of a synthetic antiferromagnet for the reference layer and a bilayer for the free layer. The multilayer composition is $\mathrm{IrMn}_{7} / \mathrm{CoFe}_{2.5} / \mathrm{Ru}_{0.8} /$ $\mathrm{CoFe}_{3} / \mathrm{MgO}_{0.7} / \mathrm{CoFe}_{1} / \mathrm{NiFe}_{5}$, where the subscript corresponds to the layer thickness in nanometer. The free layer is composed of a bilayer of $\mathrm{Ni}_{80} \mathrm{Fe}_{20}$ and $\mathrm{Co}_{90} \mathrm{Fe}_{10}$ which saturation magnetization $4 \pi M_{s}$ is estimated at 10500 Oe by a weighted sum of the bulk magnetizations. This value of the saturation magnetization was checked by vibrating sample magnetometer (VSM) and classical FMR measurements on a full sheet sample. After deposition the full stack is etched into a pillar with a $300 \mathrm{~nm}$ diameter circular cross section, and the tunnel junction has a resistance-area product of $3 \Omega \mu \mathrm{m}^{2}$. It is connected at the top to a large metallic lead and at the bottom to a narrow NiFe lead acting as a magnetic shield.

\section{A. Static characterization}

dc characterization was performed on the same experimental setup as for noise measurement. The two-points measurements are then corrected from the lead resistance determined by comparison between $V(I)$ measurements and the dynamical resistance extracted from the de-embedding procedure. The magnetoresistance ratio (MR) is about $80 \%$. Figure 1 shows the dependence of TMR and resistance as a function of bias current. The TMR decreases with bias voltage as commonly observed in polycrystalline magnetic tunnel junctions. By contrast to the antiparallel state resistance which decreases with bias current, the parallel state resistance is nearly constant with bias. Its nonsymmetric evolution with bias current is attributed to thermoelectrical effects.

The hysteresis cycle shows a coercive field of 15 Oe and a shift of the hysteresis curve of about -20 Oe typical of a slight ferromagnetic coupling between the reference and free layers through the tunnel barrier, either due to orange-peel effect or to edge dipolar coupling. Another source of magnetic coupling is direct exchange through the barrier; for epitaxially grown tunnel junctions, a crossover from ferromag- 


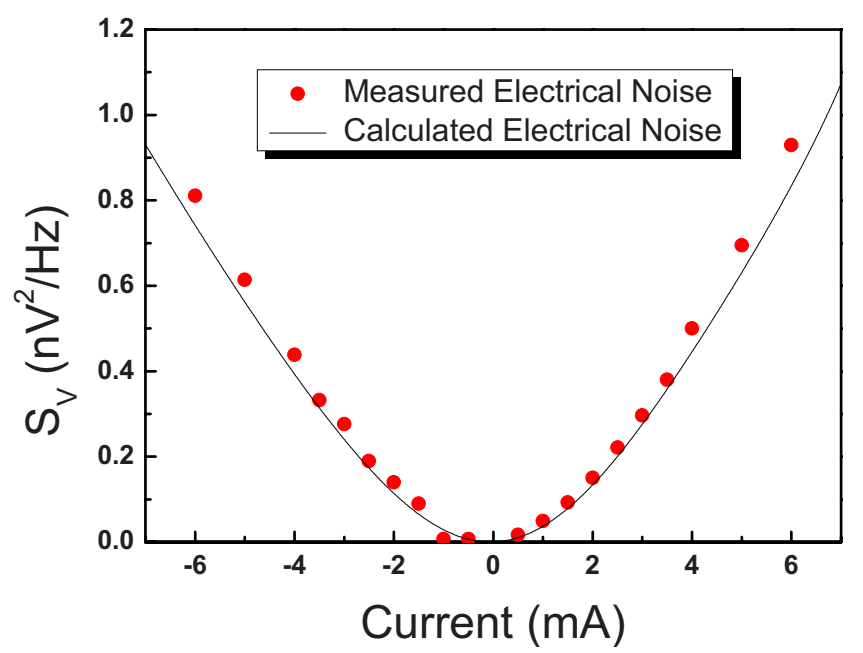

FIG. 2. (Color online) Electrical noise after subtraction of the Johnson noise as a function of the applied current in the parallel state at large field $(H=1050 \mathrm{Oe})$ when magnetic fluctuations are quenched. The solid line corresponds to the theoretical formula (24) with $R_{\mathrm{dc}}$ corresponding to the values on Fig. 1 and $R_{\mathrm{ac}}$ extracted from measurements performed with a vector network analyzer.

netic to antiferromagnetic coupling was previously reported for $0.8 \mathrm{~nm} \mathrm{MgO}$ tunnel barrier. ${ }^{16}$ However, since our samples were prepared by sputtering, the sign of the coupling cannot be directly compared with these observations.

\section{B. Electronic noise}

Electrical noise characterization due to magnetic fluctuations is reported in Sec. V. Before dealing with this subject, we first address the white background noise observed in all experiments. For that purpose a large magnetic field (1050 Oe) was applied to the junction in order to quench magnetization fluctuations. Voltage noise was then measured both in parallel and antiparallel states as a function of dc-current bias. The experimental data were corrected with the procedure described in the previous section. The only difference is that we extract here the electrical noise. Therefore the noise at zero bias is subtracted from the data to remove the background Johnson noise and the preamplifier noise. The electrical noise is plotted as a function of the applied current in Fig. 2. For perfect tunnel junctions the electrical noise is expected to follow the formula, ${ }^{17}$

$$
\left\langle v_{n}^{2}\right\rangle=2 e V \frac{R_{\mathrm{ac}}^{2}}{R_{\mathrm{dc}}} \operatorname{coth}\left(\frac{e V}{2 k T}\right),
$$

where $R_{\mathrm{dc}}=V / I$ and $R_{\mathrm{ac}}=d V / d I$. This expression describes the evolution between Johnson noise at zero bias toward shot noise at nonzero bias when $V \gg k T / e$. Since our correction procedure of experimental data requires to subtract the noise at zero bias, the corrected data are plotted considering the above expression from which the Johnson noise was subtracted for both $\mathrm{P}$ and AP states. The agreement between experiment and theory proves the quality of the tunnel junctions and the accuracy of our experimental measurements.

\section{MAGNETIC FLUCTUATIONS}

At lower magnetic field, noise spectra show peaks that are ascribed to thermally activated magnetic fluctuations at the ferromagnetic resonance. It is observed that this magnetic noise is either amplified or reduced depending on the bias current direction. This effect is interpreted as the influence of spin-transfer torque on the magnetization fluctuations and described using the model presented in Sec. II. The comparison of experimental data with the model predictions leads to a quantitative estimation of the spin torque parameters $a_{j}$ and $b_{j}$. For that purpose it is necessary to convert the voltage noise spectrum into a magnetization fluctuations spectrum. This conversion is explained in Sec. V A. Then the dependences of magnetic fluctuations on magnetic field and on current (or voltage) bias are presented.

\section{A. Data processing}

The excess voltage noise above the background noise discussed in Sec. IV B is the consequence of magnetization fluctuations through the magnetoresistive effect. So this voltage noise can be converted into magnetization noise provided the MR dependency on current bias is taken into account. Nevertheless as we deal with magnetic tunnel junctions, it is more relevant to use the magnetoconductance ${ }^{18}$ in our calculations, particularly when the MR ratio is large, $G=G_{0}+\frac{\Delta G}{2} \cos \theta$, where $\Delta G$ is the difference between the $\mathrm{P}$ state conductance and the AP state one and $\theta$ is the angle between the magnetization directions of the two layers. All measurements are performed with the applied field slightly misaligned from the reference magnetization. The misalignment angle $\theta_{0}$ is small so that $\sin \theta_{0}$ $\approx \theta_{0}$. The estimation of this angle will be extracted from the experimental data in the following. From the above formula, it is straightforward to show that the conductance fluctuations are linked to the transverse magnetization fluctuations $m_{y}$ by $\delta G=\frac{\Delta G}{2} \theta_{0} m_{y} / M_{s}$. This expression is equivalent to

$$
\delta R=\frac{\Delta R}{2 R_{\mathrm{P}} R_{\mathrm{AP}}} R^{2} \theta_{0} \frac{m_{y}}{M_{s}},
$$

where $R$ is the resistance of the junction either equal to the parallel state resistance $R_{\mathrm{P}}$ or to the antiparallel resistance $R_{\mathrm{AP}}$ depending on the bias field orientation and $\Delta R=R_{\mathrm{AP}}$ $-R_{\mathrm{P}}$. The relationship between voltage and magnetization power spectral densities is then

$$
S_{V}=I^{2} \theta_{0}^{2}\left(\frac{\Delta R}{2 R_{\mathrm{P}} R_{\mathrm{AP}}} R^{2}\right)^{2} \frac{S_{y y}}{M_{s}^{2}} .
$$

Voltage noise measurements are converted into magnetic noise spectra with the help of Eq. (26) taking into account the fact that $R, R_{\mathrm{P}}, R_{\mathrm{AP}}$, and $\Delta R$ depend on the applied current $I$. Since the misalignment angle $\theta_{0}$ is the only unknown parameter, the amplitude of the magnetic noise is determined relative to this parameter.

\section{B. Influence of the applied field}

In the normalized noise spectra shown in Fig. 3, three well-defined peaks are observed that shift toward higher fre- 


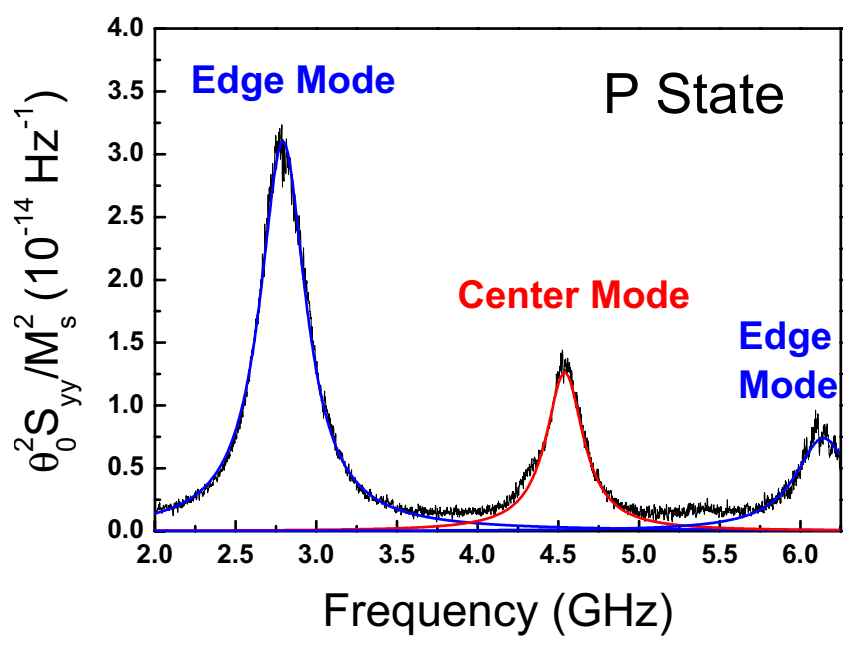

FIG. 3. (Color online) Normalized noise spectrum in the parallel state showing three distinct peaks in the measured frequency range. The second peak is attributed to the center mode whereas the two others are ascribed to edge modes. Solid lines are Lorentzian fits of the peaks.

quencies with increasing applied field. This observation is consistent with the Kittel formula as shown in Fig. 4. Since the frequencies of the peaks evolve with nearly the same slope, it is reasonable to ascribe these peaks to different spinwave modes. After comparison of the measured spectra with those obtained from micromagnetic simulations ${ }^{19}$ taking into account thermal excitations and spin-transfer torque, we ascribe the first and third peaks to first- and second-order edge modes and the second peak to the first-order center mode. This peak indexation is consistent with the fact that a slower magnetization dynamics is expected at the edges. ${ }^{20}$ Following the Kittel formula the slope of the frequency square dependence on applied field gives the saturation magnetization

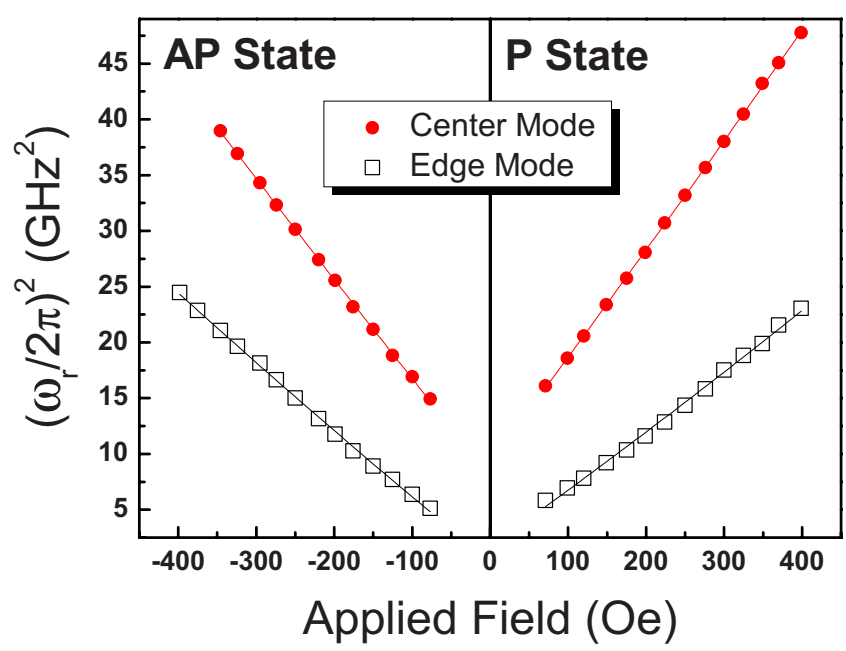

FIG. 4. (Color online) Resonance frequency versus field of the first two peaks in both the parallel and antiparallel states. The evolution of the resonance frequency with applied field is fitted with the Kittel formula $\omega_{r}^{2}=\gamma_{0}^{2}\left(4 \pi M_{s}+H\right) H$. The effective $4 \pi M_{s}$ value obtained from the fit is $6.5 \mathrm{kOe}$ for the two edge modes and 11.2 $\mathrm{kOe}$ for the center mode.
$M_{s}$. In fact FMR measurements on full sheet samples lead to an extracted value of $M_{s}$ corresponding to the bulk value. However, for the patterned tunnel junctions, the slope of the Kittel formula leads to an effective value of $4 \pi M_{s}$ due to micromagnetic effects and coupling between layers. The value obtained for the first and third peaks is much smaller than the bulk saturation magnetization whereas it is close to the bulk value for the second peak. This observation supports the peak indexation since the magnetization dynamics of the center mode is expected to be closer to the one of the bulk. Note that the slight asymmetry of the Kittel slopes between the $\mathrm{P}$ and AP states is attributed to a dynamic coupling between the free and reference layers.

As expected, the peak linewidth does not significantly vary with the applied field $\Lambda \approx \gamma_{0} \alpha M_{s}$. Knowing the saturation magnetization from the Kittel slope, the damping parameter is estimated from the linewidth value $\alpha=0.008$. Consistently with our model, the inverse of the peak amplitude varies nearly linearly with the applied field for $H>250 \mathrm{Oe}$. The slope of this linear dependence is proportional to $1 /\left(\theta_{0}^{2} \Lambda H\right)$. The previous determination of the linewidth gives a value around $1.8^{\circ}$ for the misalignment angle $\theta_{0}$. At smaller applied field, the evolution of $\theta_{0}$ with applied field is responsible for a more complex dependence of the peak amplitude as a function of the applied field. From this dependence on the applied field, it is possible to extract the misalignment angle which is estimated to vary from $\theta_{0} \approx 2.6^{\circ}$ to $1.8^{\circ}$ when the applied field varies between 100 and 250 Oe. Since $\theta_{0}$ is not precisely determined, we report $\theta_{0}^{2} S_{y y}$ in the rest of the paper.

\section{Influence of parallel spin torque}

Magnetic noise is measured at different dc-current bias in the parallel and antiparallel states for symmetric fields relative to the hysteresis cycle center so that the FMR peak stays close to the same frequency. In contrast to the field dependence of the power spectrum, the evolution with current is quite different, revealing a huge variation in the peak amplitude and of the linewidth with little frequency shift as it appears in Fig. 7. This feature is not consistent with a field effect and cannot be related to the Oersted field. The most striking feature is a pronounced asymmetry as a function of the current sign at subcritical current bias $(j<8$ $\left.\times 10^{6} \mathrm{~A} / \mathrm{cm}^{2}\right)$. The magnetic noise is either amplified or quenched depending on the bias current direction. When comparing the parallel and antiparallel states, it is observed that the effects of positive and negative currents are exchanged. In other words, this effect exhibits an asymmetry with both current and field. The asymmetry with current sign discards any explanation based on Joule effect and the asymmetry with magnetic field sign any influence of thermoelectrical effects. This observation led us therefore to suggest that spin-transfer torque reduces or enhances magnetization thermal fluctuations, depending on whether it tends to favor or disfavor the equilibrium magnetic configuration. ${ }^{8}$

When spin-transfer torque tends to favor the parallel magnetic configuration, it is observed that the magnetic fluctuations are consistently quenched in the $\mathrm{P}$ state and enhanced 
Frequency $(\mathrm{GHz})$

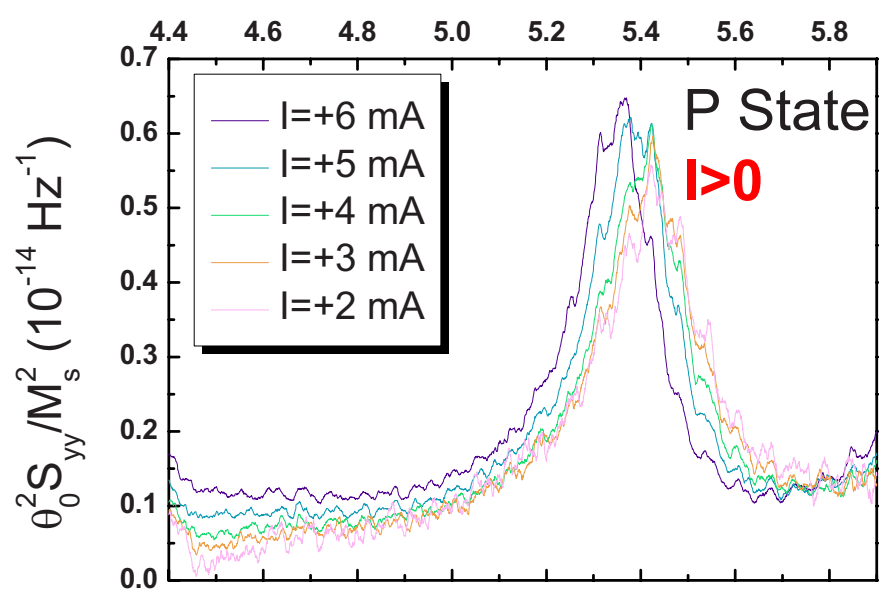

Frequency $(\mathrm{GHz})$
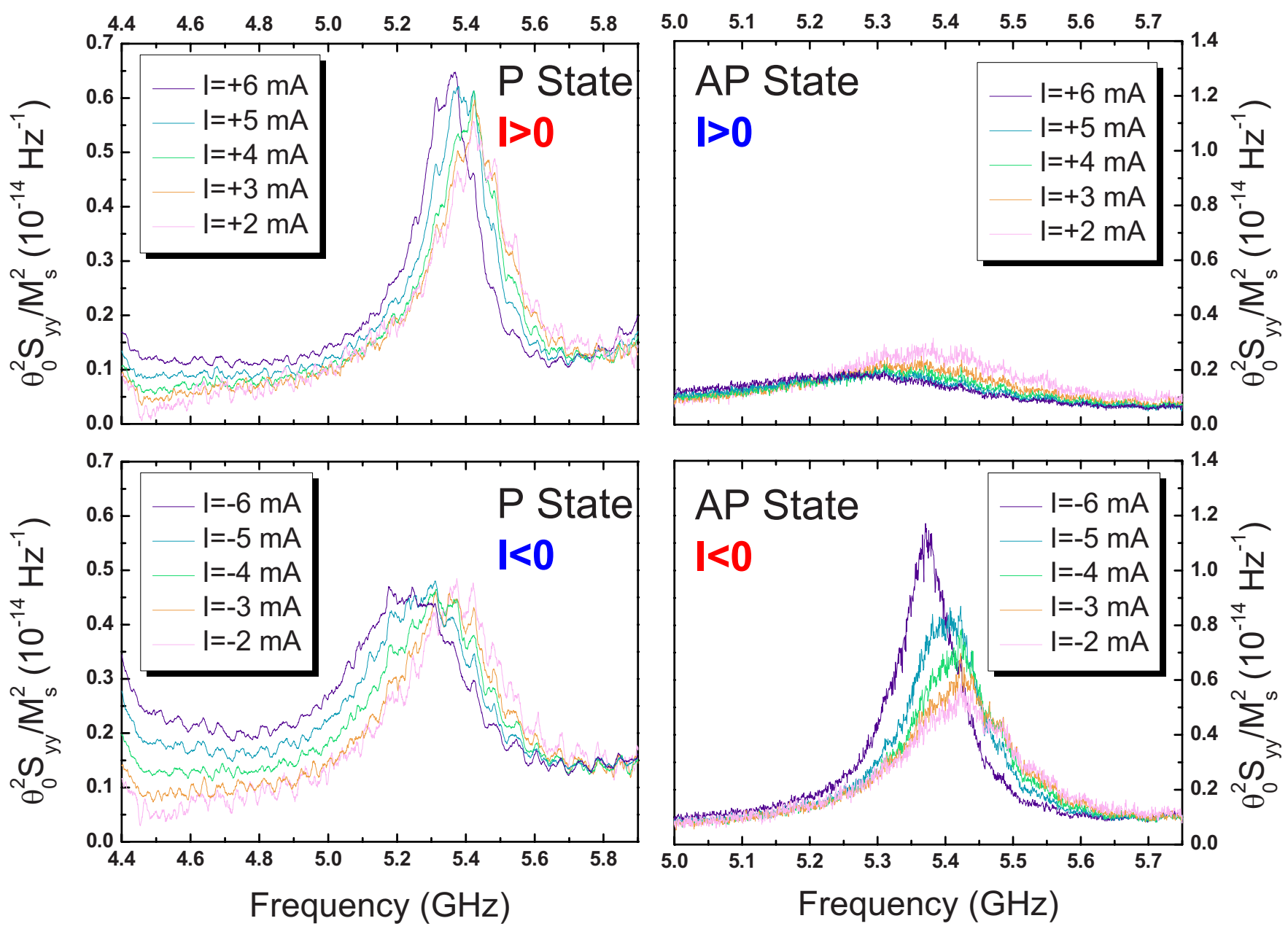

FIG. 5. (Color online) Influence of spin-transfer torque on the thermal FMR excitations for the parallel and antiparallel states. The effect is qualitatively similar on all modes. Here the center mode is represented. When peaks flatten and broaden the center mode begins to be superposed with the edge mode at low frequency. This effect appears clearly in the P state for negative currents. The peak amplitude is smaller in the P state than in the AP state which is attributed to a smaller misalignment angle $\theta_{0}$ in the P state. This interpretation is consistent with the ferromagnetic coupling observed in Sec. IV. A stronger effect of spin-transfer torque is observed in the AP state. It can be explained by the fact that a given current corresponds to a larger voltage in the AP state, therefore closer to the critical voltage (see text).

in the AP state. Moreover, when spin-transfer torque tends to favor the antiparallel magnetic configuration, its effect on magnetic fluctuations is reversed. Let us stress that the applied currents are smaller than the estimated threshold value (about $10^{7} \mathrm{~A} / \mathrm{cm}^{2}$ ), so the regions of the current-field phase diagram that are studied are subcritical states, far away from any transition to steady-state precession. In these conditions, signals are small specially at low current bias, when the voltage noise is of the order of the detection threshold. Moreover the center mode peak is particularly affected at low current bias by the FMR of the magnetic shield via the data posttreatment. The FMR of the leads is clearly observed in complex impedance measurements (see Appendix); it is responsible for an error on the transfer function. Therefore low current data were systematically discarded. Since the peak linewidth is difficult to extract with accuracy, we chose to determine it with a Lorentzian fit of the peak. On Fig. 5 the linewidth is observed to depend linearly on applied current for both the parallel and antiparallel states which is in accor- dance with the model prediction. However, the slopes in both states are different which leads to two different values of the longitudinal spin torque parameter $a=2.5 \pm 0.4$ $\times 10^{-6} \mathrm{Oe} \mathrm{A}^{-1} \mathrm{~cm}^{2}$ in the $\mathrm{P}$ state and $a=3.9 \pm 0.3$ $\times 10^{-6}$ Oe $\mathrm{A}^{-1} \mathrm{~cm}^{2}$ in the AP state. Moreover, to check the robustness of our fitting procedure, we have extracted $a$ in both $\mathrm{P}$ and $\mathrm{AP}$ states using the curve points corresponding to $-6,-5,-4,4,5$, and $6 \mathrm{~mA}$; it leads to the following results: $a=2.5 \pm 0.09 \times 10^{-6} \mathrm{Oe} \mathrm{A} \mathrm{cm}^{2}$ in the $\mathrm{P}$ state and $a$ $=3.8 \pm 0.13 \times 10^{-6}$ Oe $\mathrm{A}^{-1} \mathrm{~cm}^{2}$ in the AP state. So we can conclude that the systematic error at low current bias is not responsible for the slope difference between $\mathrm{P}$ and AP states, neither for the difference in the extrapolated critical current values. We have also checked that the error on the transfer function does not affect significantly the linewidths and the amplitudes of our spectra.

If we now plot the linewidth values as a function of the applied voltage we observe again a linear dependence of the linewidth with bias voltage but with the same slope in the $\mathrm{P}$ 

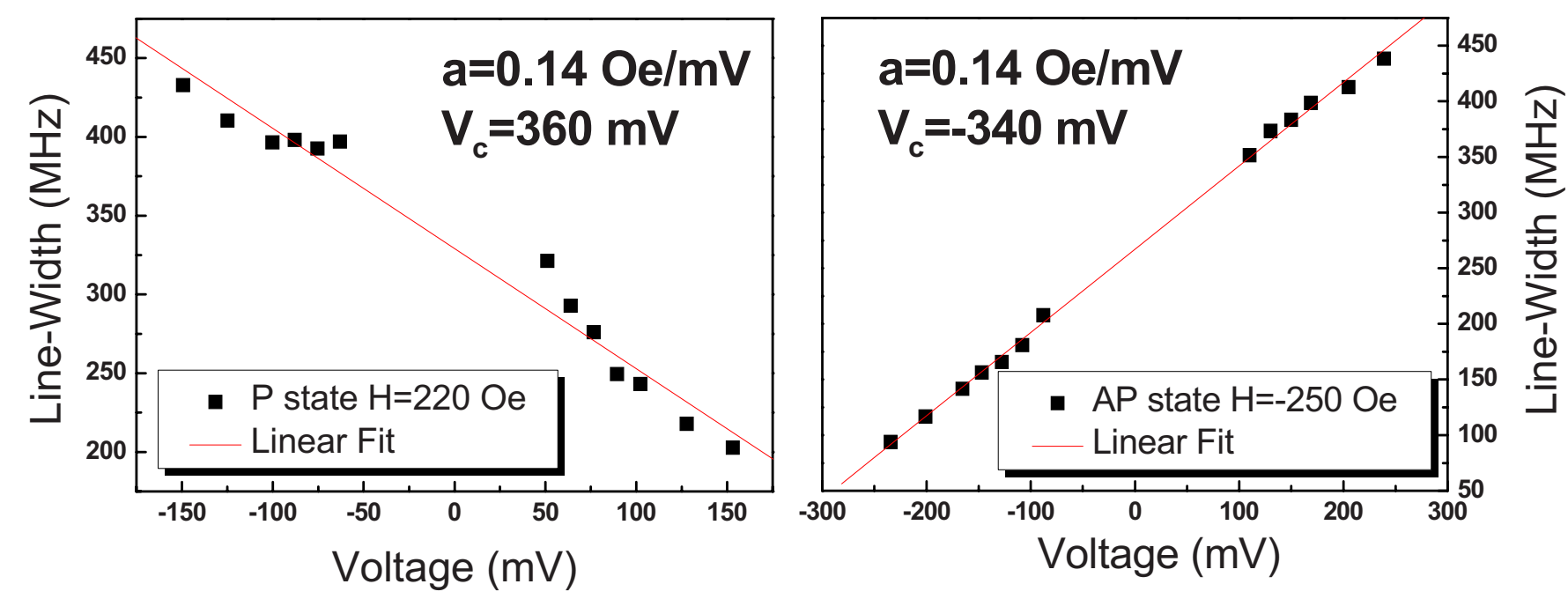

FIG. 6. (Color online) Evolution of the center mode peak linewidth with applied voltage in the parallel state (left) and antiparallel state (right). Critical voltages are estimated from the extrapolation of the linewidth to zero.

and AP state (Fig. 6). The longitudinal spin torque parameter is then $a_{j} / V=0.14 \pm 0.02 \mathrm{Oe} / \mathrm{mV}$. As discussed in Sec. II, the critical current is defined in the framework of our model as the current for which the linewidth is zero and the amplitude diverges. Therefore we define the critical bias by the extrapolation of the linewidth linear dependence to zero. The critical current estimated value is larger in the $\mathrm{P}$ state $\left(I_{c}\right.$ $=14 \pm 3 \mathrm{~mA})$ than in the AP state $\left(I_{c}=-8 \pm 1.5 \mathrm{~mA}\right)$. By contrast the estimated critical voltage is nearly the same; $V_{c}=360 \pm 80 \mathrm{mV}$ in the $\mathrm{P}$ state and $V_{c}=-340 \pm 50 \mathrm{mV}$ in the AP state. It can also be estimated from the critical current values by taking into account the resistance difference between the two states. Considering the critical voltage as the relevant parameter can explain the stronger efficiency of the spin-transfer torque observed in the AP state (see Fig. 7).

Concerning the peak amplitude, it should evolve inversely with the linewidth as discussed in Sec. II. In fact, a plot of the inverse amplitude also shows a monotonous evolution with applied current in both $\mathrm{P}$ and AP states (Fig. 8). How- ever, this dependence is linear only in the P state whereas it shows a curious nonlinear dependence in the AP state that cannot be related to the linewidth evolution. Dependences on current and on voltage are compared (Figs. 8 and 9). The critical current is again found smaller in the AP state compared to the $\mathrm{P}$ state. However, contrary to the results of the linewidth analysis, extrapolated critical voltages are not the same. Nevertheless the extrapolated critical voltages are only different by a factor 2, whereas there is a factor 3 between critical currents. The fact that there is no direct relationship between peak amplitude and linewidth contrary to the model prediction can be ascribed to the difficulty to obtain a quantitative measurement of the noise amplitude in these systems. In particular, either a potential dynamical coupling between the reference and free layers or micromagnetic effects may affect the measurement. Due to Oersted field, the micromagnetic configuration is expected to evolve with bias current and may lead to a slight change in the local misalignment angle or the local effective field. Since small variations in
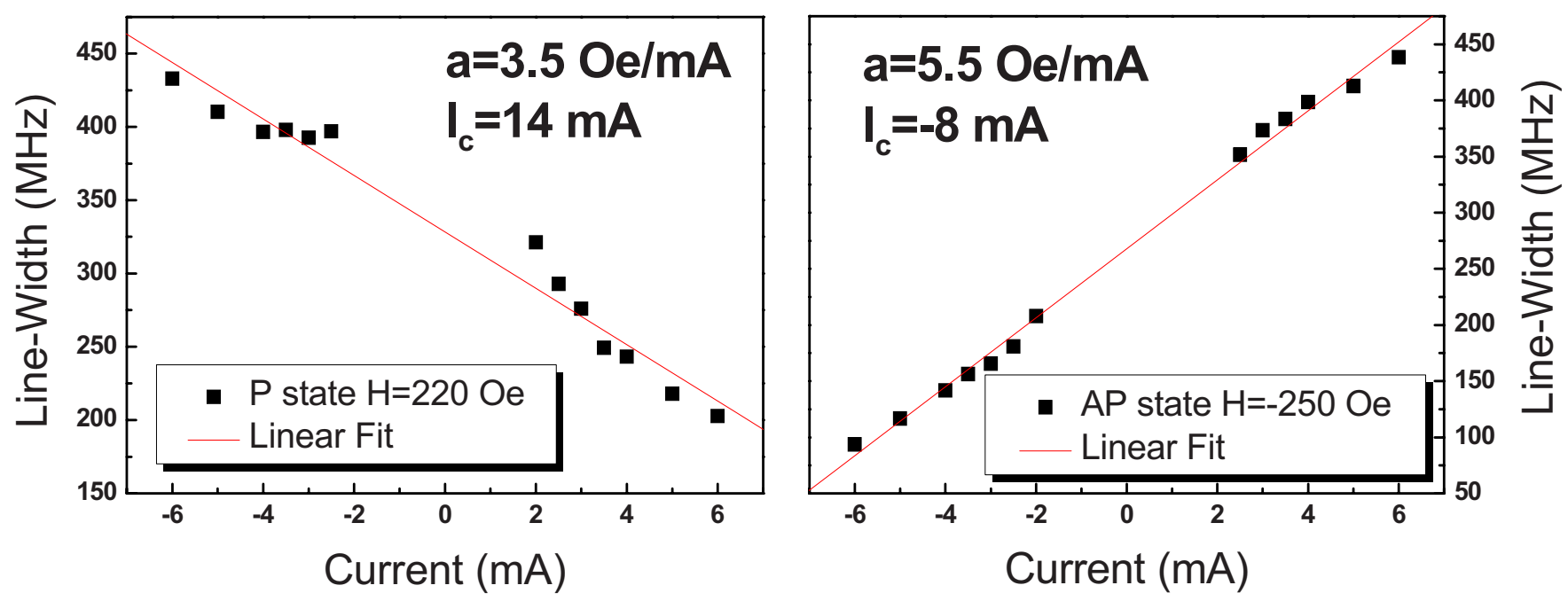

FIG. 7. (Color online) Evolution of the center mode peak linewidth with applied current in the parallel state (left) and antiparallel state (right). Critical currents are estimated from the extrapolation of the linewidth to zero. 

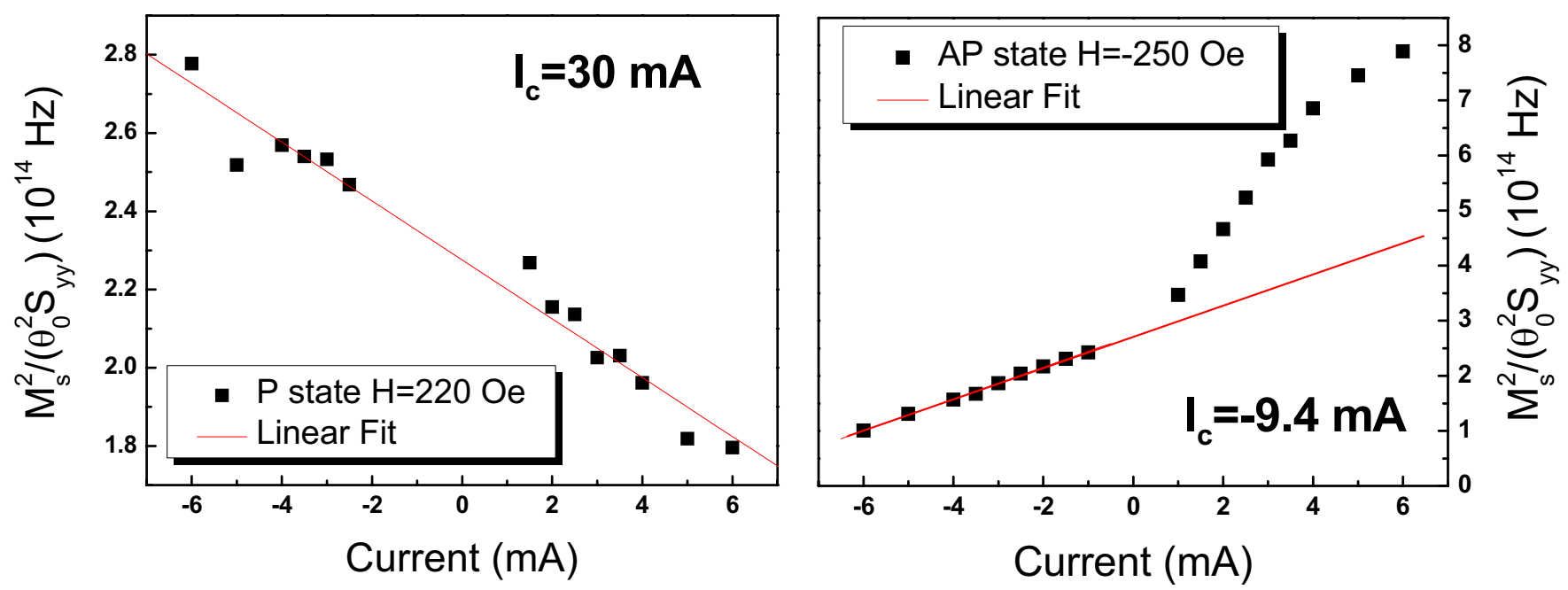

FIG. 8. (Color online) Evolution of the inverse of the peak amplitude with applied current in the parallel state (left) and antiparallel state (right). Critical currents are estimated from the extrapolation of the inverse amplitude to zero.

these parameters have a large effect on the peak amplitude, the evolution of the peak amplitude with bias current may be significantly affected. By contrast, the linewidth is less affected by these effects.

Finally let us note that linewidth and amplitude analyses give similar values of the critical current and critical voltage in the AP state. By contrast in the $\mathrm{P}$ state, the amplitude analysis leads to values of the critical current and voltage that are twice larger compared with the one extracted from the linewidth analysis. Two interesting conclusions can be drawn from this analysis. (i) Whatever the critical current estimation, currents used in the experiments are much smaller than the critical current and we a posteriori verify that we study the subcritical regime; (ii) the critical current estimated value is larger in the P state than in the AP state (Figs. 5 and 8). By contrast critical voltages are either equal when extracted from linewidth analysis (Fig. 6) or less dramatically different than critical currents when extracted from amplitude analysis (Fig. 9). This result is in agreement with
Slonczewski's prediction ${ }^{9,21}$ that the critical voltage is a more relevant parameter than the critical current in magnetic tunnel junctions.

\section{Influence of transverse spin torque}

From the model we expect to extract the second term of spin-transfer torque from the frequency shift of the magnetic noise peak. However, field and temperature ${ }^{22}$ may also affect the FMR frequency. Many artifacts such as the Oersted field, Joule heating, or thermoelectric effects have to be taken into account. But within a first-order approximation, they can be considered as independent of the magnetic configuration of the tunnel junction. Concerning Joule heating, we verified that the evolution of the FMR frequency with temperature is the same in the P and AP states. Magnetic noise measurements were then performed in both configurations by heating up the metallic sample holder and recording its temperature with a thermocouple. The FMR frequency is observed to
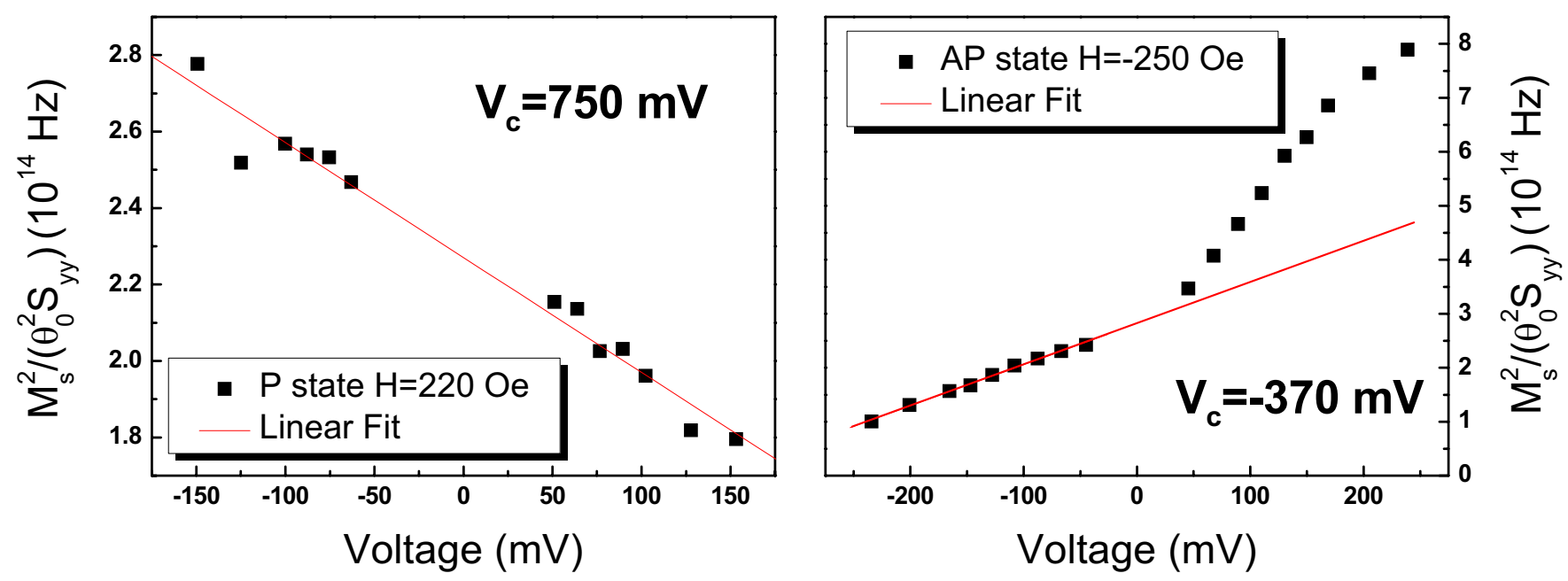

FIG. 9. (Color online) Evolution of the inverse of the peak amplitude with applied voltage in the parallel state (left) and antiparallel state (right). Critical voltages are estimated from the extrapolation of the inverse amplitude to zero. 

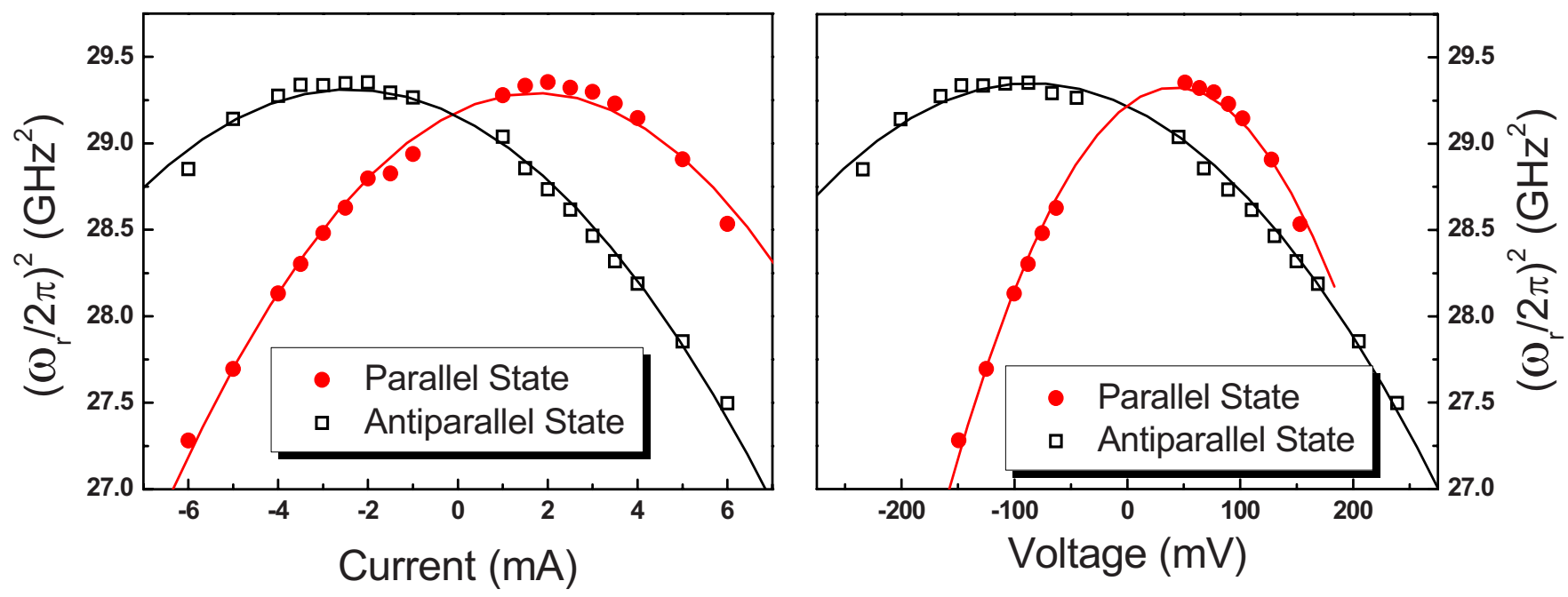

FIG. 10. (Color online) Evolution of the resonance frequency peak as a function of current and of voltage bias in both the parallel and antiparallel states. Solid lines correspond to second-order polynomial fits.

depend linearly on temperature with nearly the same slope for both configurations. In Fig. 10, the peak frequencies are plotted from the magnetic noise measurements versus bias current and voltage. It is observed that frequency decreases for both bias sign quantitatively consistent with Joule heating.

Thermoelectric effects may also be responsible for the peak frequency shift through a temperature change. They are a priori expected to be independent of the applied field and therefore of the configuration ( $\mathrm{P}$ or $\mathrm{AP}$ ) unless the change in resistance with configuration would play a significant role. However, from the asymmetric resistance changes under bias, we estimate that thermoelectric effects are small and induce the same asymmetry for both P and AP states (see Fig. 1). So we expect to remove most of the thermoelectric effects by subtraction between P and AP state.

Finally in a perfectly symmetric system, the Oersted field would have the same effect for both $\mathrm{P}$ and AP configurations under the same current bias. However, any asymmetry due to the shape, dipolar, or orange-peel coupling would lead to a different frequency shift at same current bias for the two configurations. Nevertheless we think that it is possible to suppress most of the parasitic effects due to Oersted field by subtraction between P and AP state at same current. Since the effect of Oersted field is stronger on the edges compared to the center, we expect that the center mode is less affected.

Although not perfect, this subtractive method performed at same current bias leads to a linear component of the frequency evolution which should contain the effect of spintransfer torque. Expecting that most artifacts have been removed by this method, the residual linear part can be attributed to $b_{j}$. The slope of the residual linear part is close to $1.2 \times 10^{-6} \mathrm{Oe} \mathrm{A}^{-1} \mathrm{~cm}^{-2}$. It cannot be explained by the second-order term related to $a_{j}$ as shown in Eq. (23) since $2 \alpha a_{j} \approx 0.048 \times 10^{-6} \mathrm{Oe} \mathrm{A}^{-1} \mathrm{~cm}^{-2}$. By contrast, when the subtraction is performed at same bias voltage, the resulting term shows a second-order polynomial dependence with applied voltage (see Fig. 11). The quadratic part is consistent with theoretical predictions ${ }^{23}$ and experimental spin torque
FMR measurements. ${ }^{24}$ This quadratic term is estimated to $2.2 \times 10^{-4} \mathrm{Oe} / \mathrm{mV}^{2}$ and the remaining linear term to $-0.04 \mathrm{Oe} / \mathrm{mV}$. The linear dependence obtained from subtraction at same current is partially converted in a quadratic dependence as a function of voltage since there is a large difference in resistivity between the $\mathrm{P}$ and AP states. The linear term contains the unbalanced Oersted field contribution and also a linear contribution to $b_{j}$ as discussed above. The origin of this linear contribution is not clear and may be attributed to the transport mechanism. Since the free layer is a bilayer, and due to the fact that the geometry of the junction is asymmetric, scattering may take place at the $\mathrm{CoFe} /$ $\mathrm{NiFe}$ interface as well as on defects in the barrier. These mechanisms are expected to play an important role on spintransfer torque by contrast with the pure ballistic case $\mathrm{c}^{25,26}$

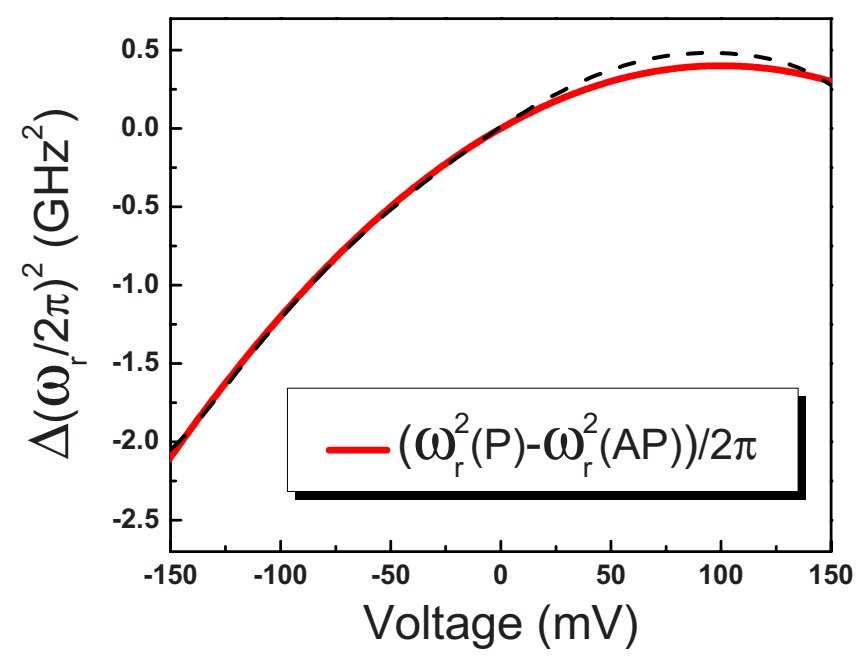

FIG. 11. (Color online) The solid line corresponds to the subtraction of the second order polynomial fits of the resonance frequency square between the P and AP states. In order to check the numerical validity of our calculation, the obtained result is compared with the subtraction of seventh order polynomial fits (dash line). 
and could be responsible for the linear term of the transverse torque term.

\section{CONCLUSION}

In this paper we have shown that subcritical spin-transfer torque influences the thermal fluctuations of the magnetization in magnetic tunnel junctions. We experimentally observed that thermally activated FMR excitations are either quenched or enhanced depending on whether the spintransfer torque favors or disfavors the thermodynamical equilibrium. This effect is described by a simple analytical model developed in the framework of the linear-response theory. It is assumed that the free layer magnetization can be described by a macrospin without coupling to the reference layer. Experimental data show a reasonably good agreement with this simple model. The parameters used in the model (the saturation magnetization $M_{s}$, the damping factor $\alpha$, the misalignment angle $\theta_{0}$, and the two spin torque term coefficients $a_{j}$ and $b_{j}$ ) are extracted independently from the magnetic noise spectrum dependencies as a function of applied field and applied current. Although the experimental noise spectra show different peaks attributed to different spin-wave modes, our simple macrospin model describes relatively well the influence of spin torque on the center mode peak. Following our model predictions, we may obtain the longitudinal torque coefficient $a_{j}$ from analysis of both the evolution of the peak linewidth $\Lambda$ and the evolution of the inverse peak amplitude with applied current. These analyses show a monotonous evolution of $a_{j}$ as predicted by the theory for small bias. However, these methods do not give the same value for $a_{j}$. The discrepancy may be attributed to the experimental uncertainty concerning quantitative amplitude measurements. Nevertheless, the critical current extracted by both methods is found smaller in the AP state compared to the P state. By contrast the obtained values correspond to the same critical voltage for both states. This result suggests that voltage is a more relevant parameter than current for describing spin-transfer torque in magnetic tunnel junctions. Finally, the transverse torque was estimated from the shift of the resonance frequency with applied bias. In spite of the difficulties of this analysis due to the extreme sensitivity of the resonance frequency to parasitic effects, we confirm the existence of a quadratic term in the transverse torque expression.

\section{ACKNOWLEDGMENTS}

Helpful discussions with M. Chshiev, J. Miltat, C. Fermon, A. Slavin, and V. Tiberkevich are gratefully acknowledged. This work was supported by the Agence Nationale de la Recherche (project SPINCHAT under Grant No. ANR-07BLAN-0341-02).

\section{APPENDIX}

This appendix describes how to obtain the transfer function which links the voltage at the MTJ and the one measured by the spectrum analyzer. To evaluate the transfer function, an electrical model of the experimental setup is necessary. A practical way is to divide it in three elements.
The first part is composed of the bias $\mathrm{T}$, the preamplifier, the cable, and the spectrum analyzer, the second corresponds to rf probe, and the last one is the sample.

\section{Electrical gain characterization}

Since the first element ends at the spectrum analyzer, it can be considered as one active dipole defined by two independent variables, its input impedance $Z_{\text {in }}$ and its gain $G$. The first step is to measure the input impedance with a vector network analyzer (VNA) as a function of frequency. The gain is measured by injecting an input voltage with a microwave source and measuring the output voltage with the spectrum analyzer. The output impedance $Z_{\text {out }}$ of the microwave source was measured beforehand in order to correct from the voltage divider. The gain measurement is performed for two different input voltages $V_{1}^{\text {sour }}$ and $V_{2}^{\text {sour }}$. This procedure eliminates the noise of both preamplifier and spectrum analyzer. The frequency dependent gain $G$ is

$$
G(\omega)=\left|\frac{Z_{\text {in }}(\omega)+Z_{\text {out }}(\omega)}{Z_{\text {in }}(\omega)}\right| \frac{V_{1}^{\text {meas }}(\omega)-V_{2}^{\text {meas }}(\omega)}{V_{1}^{\text {sour }}(\omega)-V_{2}^{\text {sour }}(\omega)},
$$

with $V_{1}^{\text {meas }}$, respectively, $V_{2}^{\text {meas }}$, as the voltage measured by the spectrum analyzer when the input voltage is $V_{1}^{\text {sour }}$, respectively, $V_{2}^{\text {sour }}$.

The second step consists in the determination of the chain matrix of the rf probe. We choose the following current convention; ${ }^{27}$

$$
\left[\begin{array}{l}
V_{1} \\
I_{1}
\end{array}\right]=\left[\begin{array}{ll}
A & B \\
C & D
\end{array}\right]\left[\begin{array}{l}
V_{2} \\
I_{2}
\end{array}\right],
$$

where $I_{1}$ is the input current into the rf probe by the contact leads, and $I_{2}$ is the output current. The determination of the four matrix elements is performed with reflexion measurements using a VNA on a calibration substrate with three calibrated loads: open, short, and $50 \Omega$ broadband load.

\section{De-embedding}

The goal of this procedure is to obtain an equivalent circuit of the sample. The sample is composed of a magnetic tunnel junction etched into a $300 \mathrm{~nm}$ diameter pillar with a top and a bottom contact leads. The magnetic tunnel junction is described by a pure real impedance $(R)$ since the capacitance of the oxide barrier can be neglected at the measured frequencies. Moreover, leads dimensions are sufficiently small compared to wavelengths involved so they can be described by discrete dipoles. The main capacitance $(C)$ stems from the facing contact leads and is represented in parallel with the pillar resistance. The description of the leads is completed by adding an inductance $(L)$ and a resistance $(r)$ in series. The equivalent circuit of the sample is given in Fig. 12.

The impedance of this equivalent circuit is then compared to the complex impedance of the sample measured with a VNA as represented in Fig. 13. The values of the capacitance, inductance, and lead resistance are deduced from the fit of the measured impedance. The obtained values $(L$ $\approx 50 \mathrm{pH}, C \approx 1.5 \mathrm{pF}$, and $r \approx 4 \Omega$ ) are in good agreement 


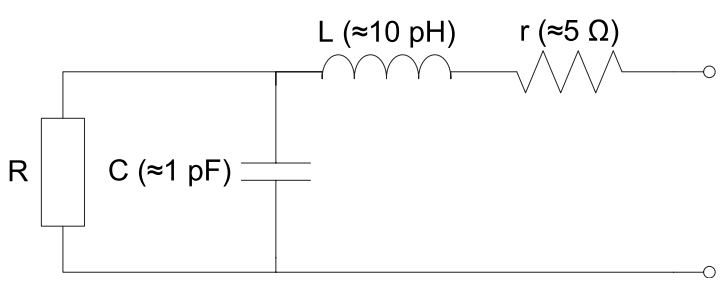

FIG. 12. Electrical description of the sample. Values indicated on the schematics are estimated from the geometry and material properties of the sample.

with the estimated values obtained by considering the leads geometry.

Note that the capacitance, composed by the facing areas between the top and bottom leads, is responsible for a reduction in the signal of $-2 \mathrm{~dB}$ at around $2 \mathrm{GHz}$.

Finally the full transfer function has the following expression:

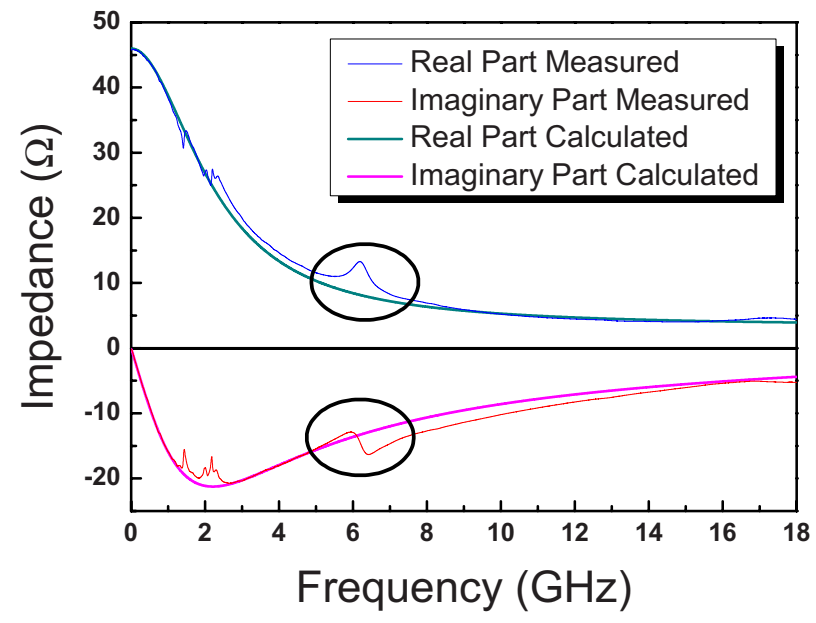

FIG. 13. (Color online) Measured and fitted impedance of the sample. The deviation observed around $5 \mathrm{GHz}$ is due to the ferromagnetic resonance of the Permalloy shield.

$$
S_{v_{m}}(\omega)=|G|^{2}\left|\frac{Z_{\text {in }}}{(1+j R C \omega)\left(A Z_{\text {in }}+B\right)} \frac{1}{[R+(1+j R C \omega)(r+j L \omega)]\left(C Z_{\text {in }}-D\right)}\right|^{2} S_{v_{s}}(\omega) .
$$

This calibration is performed for both $\mathrm{P}$ and AP state and due to the non-Ohmic behavior of the tunnel junction, this calibration procedure must be repeated for each different applied dc current. It is important to notice that the calculation of the transfer function does not allow to take into account the FMR of the Permalloy shield which appears in the measured impedance of the sample (see Fig. 13). As a consequence the error introduced by the post-treatment of raw data with the transfer function is larger around the FMR frequency of the Permalloy shield. Since the free layer and the shield are in Permalloy, their FMR frequencies are close and vary with the applied field at a similar rate, but the FMR frequency of the Permalloy shield does not much vary with applied current.
${ }^{1}$ L. Berger, Phys. Rev. B 54, 9353 (1996).

${ }^{2}$ J. C. Slonczewski, J. Magn. Magn. Mater. 159, L1 (1996).

${ }^{3}$ E. B. Myers, D. C. Ralph, J. A. Katine, R. N. Louie, and R. A. Buhrman, Science 285, 867 (1999).

${ }^{4}$ J. A. Katine, F. J. Albert, R. A. Buhrman, E. B. Myers, and D. C. Ralph, Phys. Rev. Lett. 84, 3149 (2000).

${ }^{5}$ S. I. Kiselev, J. C. Sankey, I. N. Krivorotov, N. C. Emley, R. J. Schoelkopf, R. A. Buhrman, and D. C. Ralph, Nature (London) 425, 380 (2003).

${ }^{6}$ W. H. Rippard, M. R. Pufall, S. Kaka, S. E. Russek, and T. J. Silva, Phys. Rev. Lett. 92, 027201 (2004).

${ }^{7}$ I. N. Krivorotov, N. C. Emley, J. C. Sankey, S. I. Kiselev, D. C. Ralph, and R. A. Buhrman, Science 307, 228 (2005).

${ }^{8}$ S. Petit, C. Baraduc, C. Thirion, U. Ebels, Y. Liu, M. Li, P. Wang, and B. Dieny, Phys. Rev. Lett. 98, 077203 (2007).

${ }^{9}$ J. C. Slonczewski, Phys. Rev. B 71, 024411 (2005).

${ }^{10}$ H. T. Hardner, M. B. Weissman, M. B. Salamon, and S. S. P. Parkin, Phys. Rev. B 48, 16156 (1993).

${ }^{11}$ W. F. Brown, Phys. Rev. 130, 1677 (1963).
${ }^{12}$ N. Smith, J. Appl. Phys. 90, 5768 (2001).

${ }^{13}$ N. Smith, J. Appl. Phys. 92, 3877 (2002).

${ }^{14}$ Sh. Kogan, Electronic Noise and Fluctuations in Solids (Cambridge University Press, Cambridge, 1996).

${ }^{15}$ D. C. Ralph and M. D. Stiles, J. Magn. Magn. Mater. 320, 1190 (2008).

${ }^{16}$ J. Faure-Vincent, C. Tiusan, C. Bellouard, E. Popova, M. Hehn, F. Montaigne, and A. Schuhl, Phys. Rev. Lett. 89, 107206 (2002).

${ }^{17}$ K. B. Klaassen, J. C. L. van Peppen, and X. Xing, J. Appl. Phys. 93, 8573 (2003).

${ }^{18}$ J. C. Slonczewski, Phys. Rev. B 39, 6995 (1989).

${ }^{19}$ B. Montigny and J. Miltat, J. Appl. Phys. 97, 10 C708 (2005).

${ }^{20}$ R. D. McMichael and B. B. Maranville, Phys. Rev. B 74, 024424 (2006).

${ }^{21}$ J. Z. Sun and D. C. Ralph, J. Magn. Magn. Mater. 320, 1227 (2008).

${ }^{22}$ N. Stutzke, Appl. Phys. Lett. 82, 91 (2003).

${ }^{23}$ I. Theodonis, N. Kioussis, A. Kalitsov, M. Chshiev, and W. H. 
Butler, Phys. Rev. Lett. 97, 237205 (2006)

${ }^{24}$ Jack C. Sankey, Yong-Tao Cui, Jonathan Z. Sun, John C. Slonczewski, Robert A. Buhrman, and Daniel C. Ralph, Nat. Phys. 4, 67 (2008).

${ }^{25}$ A. Manchon, N. Ryzhanova, N. Strelkov, A. Vedyayev, and B.
Dieny, J. Phys.: Condens. Matter 19, 165212 (2007).

${ }^{26}$ Peter M. Levy and Albert Fert, Phys. Rev. Lett. 97, 097205 (2006).

${ }^{27}$ D. M. Pozar, Microwave Engineering, 3rd ed. (Wiley, New York, 2005). 